\title{
Application of Taguchi method in optimising thermal comfort and cognitive performance during direct load control events
}

\author{
Fan Zhang*, Richard de Dear \\ Indoor Environmental Quality Laboratory, Faculty of Architecture, Design and Planning, The \\ University of Sydney, Australia
}

*Corresponding author:

Fan Zhang

Wilkinson Building (G04),

The University of Sydney,

NSW 2006,

Australia.

Tel.:+ 61286270929

Fax: + 61293513031

Email: fan.zhang1@sydney.edu.au

\begin{abstract}
Direct load control (DLC) is a demand response strategy that allows a utility or an aggregator to cycle specific appliances of their customers on and off or implement thermostat setback during peak demand periods. In the present research, a methodology is proposed to optimise DLC air-conditioning algorithms in order to achieve optimum and robust thermal comfort and cognitive performance outcome for commercial building occupants using Taguchi method. Human subject experiments were carried out simulating DLC events with four control factors in a university lecture theatre. Results reveal that off cycle fraction and adapting temperature are the two most influencing control factors that affect both the variability and mean response of building occupants' thermal sensation; off cycle fraction is the only significant control factor that affects the robustness of occupants' cognitive performance while none of the four control factors has any significant impact on the mean performance scores. DLC algorithms with off cycle fraction not higher than $50 \%$ and adapting temperature lower than occupants' neutral temperature are recommended to achieve optimum and robust thermal comfort and cognitive performance outcome. Cycling period and building envelope thermal performance do not have any significant impact. DLC air-conditioning strategies can be widely implemented in commercial buildings with various thermal performance conditions as long as the off cycle fraction and adapting temperature are optimised.
\end{abstract}

Keywords: Direct load control (DLC); air-conditioning; Taguchi method; thermal sensation; cognitive performance

\section{Introduction}

1.1 Direct load control of air-conditioners and its impact on thermal comfort and cognitive performance 
The increasing peak demand in the national electricity market in Australia is driven primarily by growth in air-conditioners $(A C)$, especially in residential and small commercial buildings [1]. The majority of activities aiming at reducing peak load in Australia are targeting the commercial and industrial sectors, simply because they are logistically easier to address than the residential sectors [1]. Direct load control (DLC) is a widely used demand response strategy that allows a utility or an aggregator to cycle specific appliances of their customers on and off or implement thermostat setback during peak demand periods [2]. The commonly targeted appliances in DLC programs are airconditioners, electric water heaters and pool pumps; this paper only discusses the DLC of airconditioners.

Duty cycle restriction is the most commonly used air-conditioning control approach in DLC. It involves cycling the AC compressor on and off at predetermined intervals [3]. This program maintains the thermostat setting while forcedly switches off and on the compressor according to predetermined schedules. The fan, however, will be on all the time for fresh air supply. Off cycle fraction refers to the amount of time the AC compressor will be off during an activation period [4]. Cycling period is the time for one complete cycle of AC compressor on and off [4]. A review of current DLC AC programs in USA and Australia [5-14] reveal that residential customers are the main targets addressed by current DLC programs. The duration of a DLC event usually ranges from 2 to 6 hours. Multiple off cycle fractions are usually available for customers to choose from. Yet, $50 \%$ off cycle fraction with a $30 \mathrm{~min}$ cycling period is the most commonly adopted DLC algorithm for both residential and commercial customers, meaning that $\mathrm{AC}$ is cycled on and off at $15 \mathrm{~min}$ intervals through the DLC event. Other off cycle fractions include $30 \%, 33 \%, 65 \%, 75 \%$ and $100 \%$. For $100 \%$ off cycle fraction, the AC compressor will be off for the whole duration of the DLC event. The off cycle fraction offered for commercial customers are generally lower than residential customers $[9,11,13,14]$.

Implementation of DLC air-conditioning strategies (by duty cycle restriction approach), however, may induce temperature cycles [15] in the indoor environment, and may potentially compromise building occupants' thermal comfort and cognitive performance. The authors' previous works [4, 16, 17] have selected a university lecture theatre located in Sydney as a "worst-case" scenario for DLC events given the much higher occupant density in lecture theatres than in residential buildings. The climate chamber experiments of 6 DLC air-conditioning algorithms with university student participants suggested that half of the tested DLC algorithms achieved good thermal acceptability by participants; the cognitive performance was generally unaffected by tested DLC events. Yet, the previous studies did not discuss the rationale to select DLC algorithms in order to achieve optimum and robust thermal comfort and cognitive performance outcome for building occupants.

\subsection{Robust design by Taguchi methods}

Robust design aims at a stable (robust) performance that is minimally affected by noise. Noise factors are factors that influence the performance of a system or product, but are not under control during the intended use of the product; control factors are the design parameters that need to be optimized [8]. Noise factors are the sources of variation in products and processes and therefore the cause of poor quality. The signal-to-noise ratio is a unique metric central to robust design: it allows practicing engineers to use powerful experimental analysis methods to optimize the robustness of a product or process, which can be used to identify the control factor settings that minimize the effect of noise on the response [18].

Taguchi method [19] provides a robust design for products or processes by finding control factor setpoints that will dampen the effect of the noise factors and thus reduce the variation of response from its target performance at low cost [20]. It accomplishes this goal by a two-step optimization process. During the first step, all factors that have substantial effects on the signal-to-noise ratio should be set at the levels where the signal-to-noise ratios are maximized. Then, during the second stage, the level of 
one or more factors that substantially affect the mean but not the signal-to-noise ratio should be adjusted in order to put the response on target [18].

The experimental design employed by Taguchi, known as orthogonal arrays [21], is a simple and useful tool for planning industrial experiments since it requires only a fraction of the full-factorial combinations. An orthogonal array is a matrix of numbers arranged in rows and columns. Each row represents the levels (or states) of the selected factors in a given experiment, and each column represents a specific factor whose effects on the output (or response) are of interest to the experimenters [20]. The arrays are designed to handle as many factors as possible in a certain number of runs. Orthogonal designs allow researchers/engineers to estimate the effect of each factor on the response independently of all other factors.

The Taguchi method is widely implemented in manufacturing process [22-24] and many academic domains such as environmental sciences [25, 26], agricultural sciences [27], physics [28], chemistry [29], statistics [30], management and business [31], medicine [32] etc., whenever the setpoints of factors are necessary. The application of Taguchi method in built environment domain is, however, limited in previous literature. Chlela et al. [33] adopted Taguchi method to optimize the envelope characteristics of an office building in order to improve its energy performance. Yi et al. [34] presents a methodology of environmental building design with an integrated energy-emergy approach to study building form optimization in the schematic phases. Specifically, they employed the Taguchi method to develop a metamodel for faster and easier whole building emergy simulation. Lin \& Ma [35] applied Taguchi method and Fibonacci search method to optimise phase change materials enhanced buildings with integrated solar photovoltaic thermal collectors. Verma \& Murugesan [36] employed Taguchi method and utility concept to optimise the solar collector area and ground heat exchanger length for achieving higher coefficient of performance of solar assisted ground source heat pump system.

\subsection{Aims and scopes of this paper}

Till now, Taguchi method has never been used as an optimisation strategy for DLC air-conditioning control (specifically via duty cycle restriction approach) to achieve robust and optimal thermal comfort and cognitive performance outcomes for building occupants. This study represents such an exploration of methodology to optimise DLC algorithms. To be specific, this paper aims to answer the following research questions:

1) What are the relative contributions of each control factor to building occupants' thermal comfort and cognitive performance outcome during DLC events?

2) For a certain control factor, which level of values achieves the best outcome?

3) What is the optimum combination of control factor values in order to achieve an optimum and robust thermal comfort and cognitive performance outcome during DLC events?

\section{Methods}

\subsection{Control factors and experimental design}

The authors' previous work [4] simulated a full factorial design of 48 DLC algorithms representing different thermal environments induced by three off cycle fractions $(33 \%, 50 \%$ and $67 \%)$, two cycling periods $(0.5 \mathrm{~h}$ and $1 \mathrm{~h})$, two cooling set-point temperatures before DLC events (hereinafter called adapting temperatures - the temperatures to which the human skin is adapted when the change starts; $22{ }^{\circ} \mathrm{C}$ and $24^{\circ} \mathrm{C}$ ), two building envelope thermal performance conditions (good and poor) and two ventilation rates in university lecture theatres $(10 \mathrm{~L} / \mathrm{s} /$ person and $15 \mathrm{~L} / \mathrm{s} /$ person). Since ventilation rate was found to have the smallest impact on thermal environments during DLC events compared with 4 other parameters, laboratory experiments maintained a constant ventilation rate of $10 \mathrm{~L} / \mathrm{s} /$ person, deemed typical for Australian university lecture theatres. Note that the level of values for off cycle fraction and cycling period are determined by commonly used values in previous DLC programs [5- 
14]; for adapting temperatures, $22^{\circ} \mathrm{C}$ is the common cooling setpoint temperature in buildings of Australia and $24^{\circ} \mathrm{C}$ is approximately the neutral temperature for university sedentary students dressed in typical summer clothing in Sydney. The U-value of poor building envelope thermal performance condition is 1.91 for external walls and 2.52 for roofs while the U-value of good building envelope thermal performance condition is 0.25 for external walls and 0.15 for roofs. Detailed building fabrics for simulation can be seen in [4].

To replicate simulated DLC events in a controlled environmental chamber, the orthogonal array design was applied to minimise the number of experiments, meanwhile still give the full information about all factors that affect the output. Figure 1 is a schematic diagram about the measured responses, control factors and noise factors selected for experiments to optimize participants' thermal sensation vote (TSV) and cognitive performance during DLC events. In the experimental design, subjects' thermal sensation and cognitive performance are the two measured responses (outputs) from the system. The off cycle fraction, cycling period, building envelope thermal performance and adapting temperature serve as four control factors. However, noise factors vary from different measured responses: for TSV, possible noise may come from subjects' various ages, sexes, ethnicities, metabolic rates, etc., as have been found by previous researches [16, 37, 38]; for cognitive performance, noise factors could be different development of automatic processes, motivation, skill levels of individuals, sex, etc. [17, 39]. However, it is not feasible to run orthogonal array experiments with a large number of noise factors; thus different noise factors could be grouped and compounded [18]. Given that a lot of noise factors for TSV and cognitive performance scores are person-specific factors, interpersonal difference was adopted as the compound noise factor for both measured responses.

\section{Figure 1}

Given the number of control factors, and levels of values for each factor that need to be tested, a mixed level orthogonal array of $L_{8}\left(2^{4}, 4^{1}\right)$ (Figure 2) was adopted to test a single factor (off-cycle fraction) with 4 levels and 4 other factors (cycling period, adapting temperature, building envelope thermal performance and a blank factor with 2 levels). In Figure 2, each column denotes a factor and each row represents a combination of different factors in an experiment. A two-level factor has two values: 0 and 1 , and a four-level factor has four values: 0, 1,2 and 3. Apart from the three off-cycle fractions tested in [4], $0 \%$ was a fourth level, serving as the control condition without DLC event. There was a 2-level factor deliberately left blank in order to account for experimental errors and interactive effects. Robust design assumes that the main effects are strong enough to stand out from the random noise (experimental error) and that they are stronger than interactive effects between the control factors [18] In this experimental design, interaction effects were not the research interest thus were only accounted for by a blank column. Combinations of all factors and levels of values tested in each experimental condition were listed in Table 1.

\section{Figure 2}

Table 1

\subsection{Experimental procedure}

The laboratory experiments were carried out in a controlled climate chamber in the summer of 2014. Fifty-six university-student subjects participated in these two experiments, 28 subjects for each. Each participant experienced all four conditions within his/ her experiment, including one control condition and three DLC conditions (Table 1). During the $2 \mathrm{~h}$ former experiment period for each session, participants were required to rate their thermal sensation (by ASHARE 7-point scale) every 5 minutes via a bespoke iPad application; during 8 out of 12 questionnaire intervals in every hour, they were required to do a designated online cognitive performance test ${ }^{1}$ on the computer. In order to compare

\footnotetext{
${ }^{1}$ All tests used in this study come from the public website of Cambridge Brain Sciences (CBS) Inc.
} http://www.cambridgebrainsciences.com/. 
test scores between different participants and performance test types, each participant's score was normalized using the average score of the same person on a particular cognitive performance test under the control condition (Condition 1 for Experiment 1 and Condition 8 for Experiment 2). Detailed experimental design and procedures can be found in [16, 17].

\subsection{Analysing Taguchi design}

The signal-to-noise ratio measures how the response varies relative to the nominal or target value under different noise conditions. There are four signal-to-noise ratios commonly used for static designs depending on the goal of the experiment (Table 2). In this study, subjects' TSV should be ideally targeted at " 0 " in order to ensure a neutral thermal sensation during DLC events, whereas their normalized cognitive performance scores should be maximised to ensure that DLC events have positive or at least not negative impacts on subjects' cognitive performance. TSV can have positive, zero or negative values, and "zero" is the best (ideal) value, thus for the optimization of TSV, the "nominal is best" type of S/N ratio was selected. Subjects' cognitive performance scores are obviously positive values and the larger the better, thus "larger is better" type of S/N ratio was selected.

Table 2

Taguchi design in this study was analyzed in statistical software Minitab ${ }^{\circledR} 17$ by the following two methods: response table and the Analysis of Variance (ANOVA).

\subsubsection{Response table}

The response table provides the average response outcome for each level of each factor in a design; it also reports rank and delta values that can help assess which factors have the greatest effect on the measured response of interest. Delta measures the size of the effect by taking the difference between the highest and lowest value for each response outcome. Rank orders the factors from the greatest effect (based in the delta values) to the least effect on the measured response [40].

\subsubsection{The Analysis of Variance (ANOVA)}

In the Taguchi approach, ANOVA can be applied to two forms of data: data as measured in engineering units and data that have been transformed into $\mathrm{S} / \mathrm{N}$ ratios. The significance of the individual control factors is quantified by comparing the variance between the control factor effects against the variance in the experimental data due to random experimental error and the effects of unrepresented interactions. ANOVA also enables a quantitative estimate of the relative contribution each control factor makes to the overall measured response and expresses it as a percentage. This information can be very helpful when deciding which control factors are worth further expenditure of resources to enhance product robustness [18]. The percentage contribution of the relative effect each factor has on the measured response is expressed in Equation (1) [18]:

$$
\text { Percentage contribution }=\left(\mathrm{SS}_{\text {factor }} / \text { total SS }\right) \times 100 \%
$$

\section{Results}

Datasets for TSV and cognitive performance scores were separately imported into Minitab ${ }^{\circledR} 17$ to generate response tables and ANOVA tables that are presented and analysed in the following.

\subsection{Analysing Taguchi design for thermal sensation vote}

Table 3 listed all the control factors with different levels of values in 8 experimental conditions (runs). Also, subjects' mean TSV responses were calculated by taking a grand mean of 28 subjects' 25 TSV votes within an experimental condition. The standard deviations were calculated for each subject's average TSV within an experimental condition. The $\mathrm{S} / \mathrm{N}$ ratios were calculated by the 2 nd formula in Table 2 . 
Table 4 demonstrated the response table with delta and rank information for each control factor in $\mathrm{S} / \mathrm{N}$ ratio and the mean TSV. The values reported for each level of control factor in Table 4 were calculated by averaging the corresponding values for each level of the control factor in Table 3. For example, the value " 6.535 " for Level 1 in off cycle fraction shown in Table 4 was calculated by averaging the $\mathrm{S} / \mathrm{N}$ ratios of first two runs with off cycle fraction of " $0 \%$ " in Table 3 . The Delta value " 2.763 " measures the size of the effect "off cycle fraction" by taking the difference between the highest and lowest value for the $\mathrm{S} / \mathrm{N}$ ratios in off cycle fraction. The delta values provide an estimate of the relative factor importance, similar to the information obtained from the ANOVA process. Table 4 revealed that off cycle fraction had the largest impact on reducing variability in TSV, followed by adapting temperature, cycling period and building envelope thermal performance. It is also worthwhile to compare delta values for control factors with the value for the experimental error in "blank" factor. This determines whether the main effects for each factor are stronger than the experimental error and possible interaction effects. Table 4 showed that all the four control factor effects were more pronounced. Similarly, the delta value and rank information for four control factors in the mean TSV were also calculated. The off cycle fraction had the largest impact, followed by adapting temperature, blank factor, cycling period and building envelope thermal performance. The effects of cycling period and building envelope thermal performance were weaker than the effects of experimental error and/or interaction effects.

Table 4

Table 5 demonstrated the ANOVA table for $\mathrm{S} / \mathrm{N}$ ratios and the mean TSV. To increase the sensitivity of the F-test and enhance the significance level, the sum of squares of the control factors that have a small contribution to the overall $\mathrm{S} / \mathrm{N}$ ratio and the mean TSV is often pooled to estimate the experimental error. The rule of thumb is to pool the sum of squares of up to half of the control factors which makes about half the degree of freedom available to estimate the error variance [18]. In Table 5, it was clear that off cycle fraction together with adapting temperature contributed almost $88 \%$ to the $\mathrm{S} / \mathrm{N}$ ratios. Since the cycling period and building envelope thermal performance made much smaller contribution, the sum of squares of these two control factors were pooled with the blank column to estimate the experimental error. The ANOVA revealed that adapting temperature had a significant effect on reducing variability in TSV whereas off cycle fraction was not a statistical significant factor due to its higher degree of freedom. Yet, the off cycle fraction was still the control factor which made the largest contribution to the response (45.39\%).

Table 5

ANOVA for the mean TSV revealed that although four control factors were all statistically significant, the percentage contribution of each factor to the mean TSV was distinct. Off cycle fraction and adapting temperature were the most critical factors that affected subjects' mean TSV during DLC events, contributing to $24.92 \%$ and $10.01 \%$ of TSV respectively. Yet, the contribution that cycling period (2.24\%) and building envelope thermal performance (1.23\%) had on TSV were even less than the experimental errors and interaction effects $(2.32 \%)$, which agreed with results in Table 4.

\subsection{Analysing Taguchi design for cognitive performance}

The same analysis procedure was applied to subjects' cognitive performance outcome. Table 6 listed participants' normalized mean cognitive performance scores, the standard deviations and $\mathrm{S} / \mathrm{N}$ ratios for different combinations of factor values in 8 experimental conditions. The individual cognitive performance score was normalized in such a way that the average of the two test scores for a specific participant during the control condition was set to 100; other scores of the same participant under DLC conditions were then converted pro rata according to the reference score. In each experiment run, subjects were required to finish two sets of the same eight cognitive performance tests in two hours. The mean value reported in Table 6 was the grand mean of 28 subjects' two sets of cognitive performance test scores under specific experimental conditions after normalization. Since the cognitive 
performance scores were the larger the better, the $\mathrm{S} / \mathrm{N}$ ratios were calculated by the 1 st formula in Table 2.

Table 6

Table 7 was the response table for the $\mathrm{S} / \mathrm{N}$ ratios and the mean cognitive performance scores. The delta and rank information revealed that off cycle fraction and cycling period had the largest impact on the variability in subjects' cognitive performance scores. Adapting temperature and building envelope thermal performance, however were not likely to have any significant impact on $\mathrm{S} / \mathrm{N}$ ratios since their contribution were even less than the experimental errors. For the mean cognitive performance scores, off cycle fraction was the only control factor that exerted higher impact than the experimental errors and/or interaction effects on subjects' cognitive performance scores.

Table 7

The above conclusion was further confirmed by the ANOVA test shown in Table 8. Off cycle fraction was the only control factor that has reached statistical significance after the sum of squares of adapting temperature, cycling period and building envelope thermal performance was pooled with the blank column to estimate the experimental error. The calculation of percentage contribution also confirmed that off cycle fraction was the only dominating factor affecting the variability of subjects' cognitive performance scores (80.16\%); cycling period, although ranked the second in Table 7, made only $8.32 \%$ contribution to the $\mathrm{S} / \mathrm{N}$ ratio. Adapting temperature and building envelope thermal performance made less contribution than the experimental errors, which agrees with results in Table 7. The ANOVA test of the mean cognitive performance scores demonstrated that even the first-ranked off cycle fraction was not a statistically significant control factor, making only $2.88 \%$ contribution to the mean cognitive performance scores.

Table 8

\section{Discussions}

In the two-step optimization process, the first step is to maximize $\mathrm{S} / \mathrm{N}$ ratios to minimize sensitivity to the effects of noise. A control factor plot shows average $\mathrm{S} / \mathrm{N}$ ratio for each level of each control factor. The optimum factor level setpoints are the setpoints with the highest $\mathrm{S} / \mathrm{N}$ ratio. They should give the best response with the smallest effect due to noise. The second step is to adjust the mean response onto the target response. Those control factors that affect both the $\mathrm{S} / \mathrm{N}$ ratio and the mean should be typically used to minimize the effects of noise; those affect neither the $\mathrm{S} / \mathrm{N}$ ratio or the mean can be set at the most economical values in order to keep costs low.

\subsection{Two-step optimization of TSV}

Figure 3 illustrates the factor effects plot of $\mathrm{S} / \mathrm{N}$ ratios for subjects' thermal sensation vote. It is obvious that the optimum factor setpoints are those control factor values with the highest average $\mathrm{S} / \mathrm{N}$ levels - 50\% off cycle fraction, 1.0 cycling period, good building envelope thermal performance, and $22{ }^{\circ} \mathrm{C}$ adapting temperature. However, note that only two control factors - off cycle fraction and adapting temperature had significant effect on the $\mathrm{S} / \mathrm{N}$ ratio and made substantial percentage contribution (Table 4 and 5); cycling period and building envelope thermal performance did not significantly affect $\mathrm{S} / \mathrm{N}$ ratio thus they could be adjusted to target the ideal mean TSV value or set at their most economical levels depending on their effects at the mean TSV.

Figure 3

Figure 3 also demonstrates the factor effects plot of means for TSV. Still, off cycle fraction and adapting temperature were the two most influential control factors in determining the mean TSV. The optimum factor setpoints for these two control factors were the values that have yielded a mean TSV closest to the ideal value "0", which stand for a neutral thermal sensation-0\% or 33\% off cycle 
fraction and $22{ }^{\circ} \mathrm{C}$ adapting temperature. Since $0 \%$ off cycle fraction meant no DLC events, $33 \%$ off cycle fraction were deemed as the optimum setpoint . Regarding cycling period and building envelope thermal performance, the rank information in Table 6 and percentage contributions in Table 7 both confirmed that these two control factors did not significantly affect the mean TSV.

Combining the results for $\mathrm{S} / \mathrm{N}$ ratios and means, off cycle fraction is the most influential control factor for both the $\mathrm{S} / \mathrm{N}$ ratio and the mean TSV, so the optimum factor level setpoint with the highest $\mathrm{S} / \mathrm{N}$ ratio- $50 \%$ off cycle fraction should be selected to minimize the effects of noise. However, Figure 3 revealed that a $33 \%$ off cycle fraction yielded a TSV mean that is closer to the target mean "0" than a $50 \%$ one. Thus, there is a conflict in achieving the least variability and the optimum TSV value. In practice, both $33 \%$ and $50 \%$ off cycle fractions can be deemed as optimum values for DLC algorithms. In respect to adapting temperature, the requirement of maximizing $\mathrm{S} / \mathrm{N}$ ratio and targeting the mean is consistent $-22^{\circ} \mathrm{C}$ should be selected.

Table 9 compares the effects of control factors on thermal comfort response during DLC events between the authors' previous simulation study [4] and human subject experiment. In the simulation study, it was concluded that off cycle fractions, cycling periods and cooling set-point temperatures have relatively larger influences compared to building envelope thermal performance and ventilation rates [4]. This was based on comparing the maximum operative temperature during DLC events given other factors were fixed. Laboratory experiments by Taguchi design generally agreed with this finding except the fact that cycling period made much less contribution than off cycle fraction and adapting temperature did for both $\mathrm{S} / \mathrm{N}$ ratios and mean TSV. The contribution of cycling period and building envelope thermal performance was however similar. Previous simulation study also claimed that all else being equal, DLC algorithms with shorter cycling periods have less adverse thermal comfort impacts than the longer ones [4]. But given the insignificance of cycling period in determining thermal comfort found in this study, longer cycling period can also be adopted as long as the off cycle fraction is not higher than $50 \%$ and the adapting temperature is lower than neutral. Longer cycling period (ex. 1 h) may even be beneficial from the perspective of equipment protection and maintenance. Another conclusion the simulation study has made is that in order to maintain acceptable thermal comfort for occupants, DLC algorithms must be applied judiciously and customized to the specific building, mainly referring to the building envelope thermal performance conditions. However, conclusions from experiments using Taguchi design have revealed that building envelope thermal performance is not critical in achieving thermal comfort during DLC events. For any given building, the envelope thermal performance condition is already set. This means that the condition of the building in which DLC events are carried out, whether new or old, with good or bad thermal performance, is not a critical factor in determining building occupants' thermal comfort responses if the off cycle fraction and adapting temperature have been optimised. This finding is without doubt in favour of the large-scale implementation of DLC air-conditioning strategies in commercial buildings with high occupant densities.

Table 9

\subsection{Two-step optimization of cognitive performance}

The same optimization process could be applied to cognitive performance. Figure 4 showed the factor effects plot of $\mathrm{S} / \mathrm{N}$ ratios and means for cognitive performance scores. The optimum factor setpoints for maximizing $\mathrm{S} / \mathrm{N}$ ratios were $50 \%$ off cycle fraction, $1.0 \mathrm{~h}$ cycling period, good or poor building envelope thermal performance and $22{ }^{\circ} \mathrm{C}$ adapting temperature. However, given that off cycle fraction was the only significant control factor and made $80 \%$ contribution to the $\mathrm{S} / \mathrm{N}$ ratio (Table 8 ), the off cycle fraction should be set at $50 \%$ where it achieved the highest $\mathrm{S} / \mathrm{N}$ ratio, while other control factors could all be set at economical values.

Figure 4 
As for the means, since none of the four control factors had a significant impact on subjects' mean cognitive performance scores (Table 8), the factors could all be set at their economical values. The authors' previous work [17] adopted multilevel linear models to test the correlation between subjects' cognitive performance with different DLC conditions after the adjustment of confounding variables and results revealed that DLC events, generally, did not significantly affect participants' scores on 8 cognitive performance tests. Findings in this paper corroborated with the previous conclusions in confirming that DLC events would not adversely affect building occupants' cognitive performance. For the purpose of reducing variability in subjects' cognitive performance scores between different people, it was suggested that $50 \%$ off cycle fraction should be adopted for DLC algorithms while other factors could be set at their economical values.

To summarize the two-step optimization results, $50 \%$ off cycle fraction and a lower-than-neutral adapting temperature (ex. $22^{\circ} \mathrm{C}$ ) incurred the least variability in subjects' thermal sensation votes; $33 \%$ off cycle fraction and $22{ }^{\circ} \mathrm{C}$ adapting temperature lead to the most ideal thermal sensation close to neutral. As for cognitive performance, the adoption of $50 \%$ off cycle fraction yielded the least sensitivity to the noise; none of the control factors had any significant impact on subjects' cognitive performance scores. To make a generalisation, DLC air-conditioning algorithms with off cycle fraction not higher than $50 \%$ and adapting temperature lower than building occupants' neutral temperature are critical in achieving optimum thermal comfort and cognitive performance outcome in densely populated commercial buildings; either longer or shorter cycling period can be adopted; the envelope thermal performance of the buildings will not have any significant impact on building occupants' thermal sensation or cognitive performance during DLC events. However, since the orthogonal array adopted in this study only allowed two different levels of values in adapting temperature, it was impossible to tell how much an ideal adapting temperature should deviate from the neutral temperature in the cooler side. This research question can be addressed by future studies.

\section{Conclusions}

Taking a university lecture theatre in Sydney as an example, this study applied the Taguchi method to optimise direct load control air-conditioning strategies in the aim of obtaining optimum and robust thermal comfort and cognitive performance outcome for occupants in densely populated commercial buildings. The following conclusions can be drawn:

- For thermal sensation, off cycle fraction and adapting temperature are the two most influencing control factors for both the variability and mean response. Cycling period and building envelope thermal performance do not have any significant impact on either sensitivity to noise effect or the mean response for thermal sensation.

- For cognitive performance, off cycle fraction is the only significant control factor that affects the robustness of cognitive performance scores. None of the four control factors have any significant impact on the mean cognitive performance scores. This finding further supports the authors' previous conclusion that cognitive performance is stable across a range of thermal conditions and generally unaffected by direct load control events.

- DLC algorithms with off cycle fraction not higher than $50 \%$ and adapting temperature lower than building occupants' neutral temperature are crucial in achieving optimum and robust thermal comfort and cognitive performance for commercial buildings with high occupant densities. Neither the cycling period nor the building envelope thermal performance makes any significant impact. DLC air-conditioning strategies can be widely implemented in commercial buildings with a variety of building envelope thermal performance conditions as long as the off cycle fraction and adapting temperature are optimised.

\section{Acknowledgement}


This research is supported by University of Sydney-China Scholarship Council Postgraduate Research Scholarship and approved by the Human Research Ethics Committee at the University of Sydney (Project No. 2014/034). The authors would like to thank Dr Christhina Candido from the University of Sydney’s Indoor Environmental Quality Laboratory for her advice on the structure of this paper.

\section{References}

[1] George Wilkenfeld \& Associates Pty Ltd. A national demand management strategy for small air conditioners: the role of the National Appliance and Equipment Energy Efficiency Program (NAEEEP), Report No. 2004/22. Prepared for the National Appliance and Equipment Energy Efficiency Committee (NAEEEC)/ Australian Greenhouse Office; 2004.

[2] Albadi MH, El-Saadany EF. A summary of demand response in electricity markets. Electric Power Systems Research 2008; 78(11): 1989-96.

[3] Weller GH. New wave of direct load control update on DLC systems. http://www.elp.com/articles/powergrid international/print/volume-16/issue-7/features/new-waveof-direct-load-control-update-on-dlc-systems-technology.html, accessed 17-09-2016.

[4] Zhang F, de Dear R. Thermal environments and thermal comfort impacts of direct load control airconditioning strategies in university lecture theatres. Energy and Building 2015;86:233-42.

[5] BGE. Baltimore Gas \& Electric Co. PeakRewards ${ }^{\mathrm{SM}} \mathrm{A} / \mathrm{C}$ program. http://bgesavings.com/programs/ac, accessed 17-09-2016.

[6] ComEd. ComEd Smart Ideas ${ }^{\circledR}$ Central AC Cycling Program. https://www.comed.com/homesavings/energy-management/Pages/central-ac-cycling.aspx, accessed 17-09-2016.

[7] Con Edison. Con Edison Control your A/C remotely with a smart thermostat program. http://www.coned.com/energyefficiency/free_thermostat.asp, accessed 17-09-2016.

[8] FirstEnergy. FirstEnergy Easy Cool Rewards Program. http://energysaveohio-home.com/easy-coolrewards/, accessed 17-09-2016.

[9] FPL. Florida Power \& Light Company On Call ${ }^{\circledR}$ Program. https://www.fpl.com/save/programs/oncall.html, accessed 17-09-2016.

[10] Idaho Power. Idaho Power A/C Cool Credit Program. https:/www.idahopower.com/energyefficiency/Residential/Programs/ACCoolCredit/default.cfm, accessed 17-09-2016.

[11] PG \& E. Pacific Gas \& Electric Company SmartAC ${ }^{\mathrm{TM}}$ Program. http://www.pge.com/en/myhome/saveenergymoney/plans/smartac/index.page?WT.mc_id=Vanity smartac, accessed 17-09-2016.

[12] PSE \& G. Public Service Electric and Gas Cool Customer Program. https://www.pseg.com/business/small_large_business/save_energy/cool_customer/faq.jsp, accessed 17-09-2016.

[13] SCE. Southern California Edison Summer Discount Plan. https:/www.sce.com/wps/wcm/connect/1114de14-c46e-4921-bf16b88a5ac6610a/SDP+FACT+Sheet+NR-587-V6-0413.pdf?MOD=AJPERES, accessed 17-09-2016.

[14] SDG \& E. San Diego Gas \& Electric Summer Saver Program. http://www.sdge.com/savemoney/summer-saver-program, accessed 17-09-2016.

[15] ASHRAE. ANSI/ASHRAE Standard 55-2013. Thermal environmental conditions for human occupancy. Atlanta: American Society of Heating, Refrigerating and Air-Conditioning Engineers, Inc; 2013.

[16] Zhang F, de Dear R, Candido C. Thermal comfort during temperature cycles induced by direct load control strategies of peak electricity demand management. Building and Environment 2016;103: 9-20.

[17] Zhang F, de Dear R. University students' cognitive performance under temperature cycles induced by direct load control events. Indoor Air 2016; Article in press. doi:10.1111/ina.12296. 
[18] Fowlkes WY, Creveling CM. Engineering Methods for Robust Product Design: Using Taguchi Methods in Technology and Product Development. Pearson Education; 2012.

[19] Taguchi G. Introduction to quality engineering — designing quality into products and processes. Asian Productivity Organization; 1986.

[20] Antony J, Kaye M. Experimental Quality: A strategic approach to achieve and improve quality. Springer, US; 2012.

[21] Rao CR. Factorial experiments derivable from combinatorial arrangements of arrays. Journal of the Royal Statistical Society 1947; Suppl. 9: 128-39.

[22] Pang JS, Ansari MNM, Zaroog OS, Ali MH, \& Sapuan, SM. Taguchi design optimization of machining parameters on the $\mathrm{CNC}$ end milling process of halloysite nanotube with aluminium reinforced epoxy matrix (HNT/Al/Ep) hybrid composite. HBRC Journal 2014; 10(2): 138-44.

[23] Ghani JA, Choudhury IA, \& Hassan HH. Application of Taguchi method in the optimization of end milling parameters. Journal of Materials Processing Technology 2004; 145(1): 84-92.

[24] Pander A, Hatta A, \& Furuta H. Optimization of catalyst formation conditions for synthesis of carbon nanotubes using Taguchi method. Applied Surface Science 2016; 371: 425-435.

[25] Daneshvar N, Khataee AR, Rasoulifard MH, Pourhassan M. Biodegradation of dye solution containing Malachite Green: Optimization of effective parameters using Taguchi method. Journal of Hazardous Materials 2007; 143(1-2): 214-19.

[26] du Plessis BJ, de Villiers GH. The application of the Taguchi method in the evaluation of mechanical flotation in waste activated sludge thickening. Resources, Conservation and Recycling 2007; 50(2): 202-10.

[27] Tasirin SM, Kamarudin SK, Ghani JA, Lee KF. Optimization of drying parameters of bird's eye chilli in a fluidized bed dryer. Journal of Food Engineering 2007; 80(2): 695-700.

[28] Wu CH, Chen WS. Injection molding and injection compression molding of three-beam grating of DVD pickup lens. Sensors and Actuators A: Physical 2006; 125(2): 367-75.

[29] Houng JY, Liao JH, Wu JY, Shen SC, Hsu HF. Enhancement of asymmetric bioreduction of ethyl 4-chloro acetoacetate by the design of composition of culture medium and reaction conditions. Process Biochemistry 2007; 42(1): 1-7.

[30] Romero-Villafranca R, Zúnica L, Romero-Zúnica R. Ds-optimal experimental plans for robust parameter design. Journal of Statistical Planning and Inference 2007; 137(4): 1488-95.

[31] Elshennawy AK. Quality in the new age and the body of knowledge for quality engineers. Total Quality Management and Business Excellence 2004; 15(5-6): 603-14.

[32] Ng EYK, Ng WK. Parametric study of the biopotential equation for breast tumour identification using ANOVA and Taguchi method. Medical and Biological Engineering and Computing 2006; 44(1-2): 131-39.

[33] Chelela F, Husaunndee A, Riederer P, Inard C. A statistical method to improve the energy efficiency of an office building. In: Proceedings of international building performance simulation association conference; 2007; 1756-64.

[34] Yi H, Srinivasan, RS, \& Braham, WW. An integrated energy-emergy approach to building form optimization: Use of EnergyPlus, emergy analysis and Taguchi-regression method. Building and Environment 2015; 84: 89-104.

[35] Lin W, \& Ma Z. Using Taguchi-Fibonacci search method to optimize phase change materials enhanced buildings with integrated solar photovoltaic thermal collectors. Energy 2016; 106: 23-37.

[36] Verma V, \& Murugesan K. Optimization of solar assisted ground source heat pump system for space heating application by Taguchi method and utility concept. Energy and Buildings 2014; 82: 296-309.

[37] Fanger PO. Thermal comfort. Analysis and applications in environmental engineering. New York: McGraw-Hill; 1972.

[38] Parsons K. Human Thermal Environments: The effects of hot, moderate, and cold environments on human health, comfort, and performance (3rd Edition). Taylor \& Francis; 2014. 
[39] Hancock PA, Vasmatzidis I. Effects of heat stress on cognitive performance: the current state of knowledge. International Journal of Hyperthermia 2003; 19: 355-72.

[40] Minitab 17 Support. Two-step optimization for Taguchi designs. http://support.minitab.com/enus/minitab/17/topic-library/modeling-statistics/doe/taguchi-designs/two-step-optimization-fortaguchi-designs/, accessed 01-08-2016. 